\title{
LOS ANÁLISIS DE LA POLÍTICA EXTERIOR COLOMBIANA:
} UN ESTADO DEL ARTE

\author{
THE ANALYSIS OF COLOMBIAN FOREIGN POLICY: \\ A STATE OF THE ART
}

\author{
Carlos Hernán González Parias ${ }^{1}$ \\ ORCID: 0000-0001-6129-8662 \\ Juan Camilo Mesa Bedoya ${ }^{2}$ \\ ORCID: 0000-0001-8495-9988
}

\section{RESUMEN}

El artículo realiza una revisión de literatura académica sobre el análisis de la política exterior colombiana producida en las dos últimas décadas (2000-2019), por lo tanto, es un estado del arte. Fueron varios objetivos los que orientaron la realización de este, entre ellos: determinar las tendencias actuales, posibles vacíos y el nivel de actividad de esta línea de investigación, e identificar tanto las metodologías utilizadas como las orientaciones teóricas de dichos análisis. Esta revisión permite concluir, entre otros asuntos, que los trabajos de política exterior colombiana son una línea de análisis dinámica y activa, en la que se ha superado la etapa descriptiva y de generalizaciones, lo que ha dado paso a orientaciones teórico-metodológicas más abarcadoras, para el estudio de situaciones específicas, y a una mayor profundidad en los factores explicativos.

Palabras clave: política exterior; Colombia; posconflicto; actores estatales; actores no estatales.

\begin{abstract}
This article is a review of academic literature on the analysis of Colombian foreign policy produced in the last two decades (2000-2019), therefore, it is a state of art. There were several objectives that guided the realization of the same, including the following: determine the current trends, possible research gaps and the level of activity of this line of research, and identify the methodologies used as well as the theoretical orientations of these analyzes. It is concluded that Colombian foreign policy studies are a line of dynamic and active research, which has passed the stage of mere description and generalizations, which has given way to broader theoretical-methodological orientations for the analysis of specific situations and a greater depth in the explanatory factors.
\end{abstract}

Keywords: foreign policy; Colombia; postconflict; state actors; non-state actors.

1 Institución Universitaria Esumer, Departamento de Investigaciones Académicas, Medellín, Colombia. Docente a tiempo completo y Candidato a doctor en Ciencias Sociales por la Universidad Pontificia Bolivariana de Medellín. Correo electrónico: carlosheg@gmail.com

2 Institución Universitaria Esumer, Departamento de Investigaciones Académicas, Medellín, Colombia. Docente a tiempo completo y Candidato a doctor en Relaciones Internacionales por la Universidad de la Plata, La Plata, Argentina. Correo electrónico: mesabedoya@gmail.com 


\section{Introducción}

La política exterior colombiana, como objeto de análisis sistemático de la academia del país, se inicia en la década de 1980, en buena medida, en respuesta a la diversificación de la agenda exterior de la nación, "siguiendo las tendencias regionales de ese período, lo cual planteó la necesidad de desarrollar herramientas analíticas y prácticas para orientar el nuevo papel que el país aspiraba desempeñar en el mundo" (Tickner y Sandra, 2011, p. 22).

Sin el ánimo de desconocer publicaciones en décadas y años anteriores, sobre temáticas relativas a la política exterior, como, por ejemplo: historia diplomática, integración regional, inserción económica internacional de Colombia, es el profesor austriaco Gerhard Drekonja Kornat el considerado como el precursor de esta línea de estudio. Su libro Retos de la política exterior colombiana (1983), precisamente, es apreciado como la obra fundacional de esta vertiente investigativa, al realizar un análisis riguroso y sistemático sobre la política exterior del país.

Entre los aportes de la obra de Drekonja, se destacan: desarrollar las categorías de bajo y alto perfil de una política exterior; identificar debilidades de la política exterior colombiana (carencia de una política exterior clara, rol poco relevante del Ministerio de Relaciones Exteriores, poca capacidad negociadora y baja actividad internacional); caracterizar los aspectos relevantes de esta política en Colombia (alta juridicidad, énfasis de la acción colectiva en organizaciones regionales y globales, no intervención en asuntos de otros Estados y búsqueda de solución pacífica de diferencias internacionales). Pero la influencia más recordada del profesor austriaco es la de identificar y explicar dos tradiciones bajo las cuales, en diferentes etapas y contextos históricos, se ha orientado la conducción de la política exterior del país: respice polum y respice similia3. Ambas cuestiones, es decir, la publicación y la clasificación, aun generan influencia en los estudios en política exterior colombiana.

Posteriormente, bajo la influencia de este primer trabajo, Rodrigo Pardo y Juan Gabriel Tokatlian, publican Política exterior colombiana: ¿de la subordinación a la autonomía? (1988), texto en el cual reflexionan sobre diversas temáticas,

3 Estos dos términos fueron acuñados por ministros de relaciones exteriores, quienes, posteriormente, serían presidentes de la República: respice polum, por Marco Fidel Suárez, a principios del siglo XX, y respice similia, por Alfonso López Michelsen, finalizando la década de 1960. El primero de estos conceptos lleva a orientar la política exterior, mirando hacia la estrella del norte (Estados Unidos), y el segundo, a conducirla hacia los Estados vecinos y semejantes; "en particular que buscaban una mayor diversificación diplomática, política y económica y la afirmación de una progresiva solidaridad Sur-Sur" (Tokatlian, 2000) 
tales como la relación en Colombia entre política interna y externa, el rol del país en las problemáticas que vivía Centroamérica en la década de los 80 y una lectura de la política exterior colombiana ligada al vínculo con los Estados Unidos y América Latina.

Otro aporte representativo, de la etapa germinal, por destacar es el trabajo de Diego Cardona (1992), quien realiza un análisis de la producción académica que de alguna forma aborda el tema de política exterior colombiana durante los años 70 y 80 del siglo XX, con el objetivo de identificar las concepciones teóricas, tendencias, posiciones y enfoques adoptados, así como vacíos investigativos en dicha producción. El autor señala la ausencia casi total de estudios sobre tópicos como el proceso de toma de decisiones en la política exterior del país, los diferentes actores que inciden en la formulación y las empresas transnacionales.

Desde inicios del siglo XXI, esta línea de investigación ha contado con un importante desarrollo liderado por investigadores como Arlene Tickner (2003, 2007, 2011), Sandra Borda (2011, 2014), Socorro Ramírez (2005, 2011), Martha Ardila (2005), Roberto González Arana (2004, 2015) y Eduardo Pastrana (2011). Ellos han abordado la política exterior desde varios ángulos, entre los cuales se hallan el contexto internacional y su influencia en las directrices de la política exterior; los factores asociados al conflicto; las élites políticas colombianas; las nuevas tendencias en materia de las relaciones internacionales y en factores clave como la inserción económica; la influencia de visiones no tradicionales en la delimitación de la política exterior.

Entre los estudios realizados en los últimos años con respecto a la política exterior colombiana, se encuentran aquellos que abordan el tema de rupturas y continuidades de esta política entre los Gobiernos de Álvaro Uribe y Juan Manuel Santos; también, los estudios que analizan características, estrategias y objetivos de la política exterior de ambas administraciones de Juan Manuel Santos (2010-2018). Más recientemente, a causa del contexto histórico que atraviesa el país por la finalización del proceso de negociación entre el Gobierno nacional y las Farc, empieza a ser importante, en un sentido prospectivo, la literatura académica que versa sobre la política exterior de cara al posconflicto: agenda, retos, aliados, proyección, objetivos son algunos de los aspectos que discuten los autores aludidos.

Es trascendental mencionar que el posacuerdo genera una serie de retos en materia de política exterior, pues es innegable que el país percibe cambios que serán trascendentales para lograr la estabilidad. Por ello, los puntos clave en asuntos de desarrollo estarán enfocados en temas tan sensibles como la seguridad y la defensa; el sector extractivo en Colombia; drogas y mercado ilícito 
de armas; derechos humanos; relaciones multilaterales; comercio internacional; entre otros asuntos vitales para el Estado colombiano.

Este artículo tiene por finalidad realizar un estado del arte de la investigación alrededor de la política exterior colombiana, con base en la producción científica entre los años 2000 y 2019. En ese sentido, busca identificar tendencias, enfoques, marcos teóricos, nivel de dinamismo y posibles vacíos en torno a esa línea investigativa.

El estado del arte en sí mismo es una metodología de investigación documental (Gómez, Galeano y Jaramillo, 2015), en palabras de Martín Retamozo: “el estado de la cuestión mantiene una tensión entre mostrar que el tema fue tratado por otros autores $-y$ que se conocen sus aportes- $y$, a la vez, evidenciar que hay algo aún por decir/conocer con respecto al tema" (2014, p. 185). Los criterios de búsqueda y selección de lo producido bibliográficamente se basaron en lo siguiente: libros y capítulos exclusivamente considerados como resultados de proyectos de investigación; artículos en revistas científicas nacionales e internacionales, especializadas tanto en relaciones internacionales como en ciencias políticas, indexadas en mínimo cuatro índices bibliográficos y en acceso abierto (Open Access).

\section{Perfil y rasgos generales de la política exterior colombiana}

A lo largo del tiempo, la política exterior colombiana ha sido perfilada bajo diferentes características, por ejemplo, ser de un estilo proamericana y poseer un fuerte rasgo presidencialista, lo que conllevaría un proceso cerrado en la toma de decisiones, transitar entre el respice polum y el respice similia, así como un tradicional apego al derecho internacional para la solución pacífica de controversias, por mencionar los rasgos más recurrentes.

Sin embargo, en los últimos años, han surgido investigaciones que, dejando de lado el método descriptivo, buscan profundizar, abordar desde nuevas ópticas o cuestionar algunos de estos rasgos tradicionales de la política exterior del país. Uno de los aspectos característicos de la política exterior colombiana son los estrechos vínculos con los Estados Unidos. Esta relación ha gozado de estabilidad y armonía a lo largo del tiempo, desde el restablecimiento, por medio del Tratado Urrutia-Thomson, de los maltrechos vínculos bilaterales a causa de la pérdida de Panamá y pese a algunos episodios como el proceso 8000 . Fruto de diversas investigaciones en esta dirección, han surgido denominaciones tales 
como el tradicional respice polum (Drekonja, 1983), subordinación (Pardo y Tokatlian, 1988), intervención por invitación (Tickner, 2007) y dependencia estructural (González, Galeano y Trejos, 2015).

Precisamente, José Luis Bernal y Arlene Tickner (2017) procuran responder el porqué de la persistencia en el tiempo del proamericanismo en la política exterior colombiana y cuál ha sido su papel como elemento identitario dominante en el imaginario de esa política en el país (IPE). El análisis es centrado en las élites políticas nacionales y entendiendo imaginarios de política exterior (IPE), bajo la orientación teórica del constructivismo crítico pospositivista, como

un entramado de significados y de relaciones sociales a partir del cual los voceros del Estado construyen representaciones del mundo [...] emerge de posiciones intersubjetivas específicas e interpretaciones existentes acerca de la política mundial y es a la vez recreado por estas (Bernal y Tickner, 2017, p. 5).

En ciencias sociales, todas las "verdades", por arraigas y aceptadas que estén en la tradición académica especializada, son sujetas a ser revaluadas y revisadas, incluso como algo necesario para el desarrollo investigativo. Precisamente, Rodrigo Amaya (2017) reflexiona sobre la pertinencia de repensar algunas características que tradicionalmente le han atribuido a la política exterior del país: el excesivo presidencialismo, la proximidad a Estados Unidos y el hermetismo decisorio. Lo anterior, tratando "poner en tela de juicio y la precisión de las nociones imperantes [...] para identificar posibles alternativas que eleven nuestra comprensión del asunto" (Amaya, 2007, p. 43). Como resultado de su investigación, considera necesario subir un escalón más en los análisis de política exterior colombiana; superar lo descriptivo, lo general y las visiones monoteóricas, en procura de lograr diagnósticos estructurales, estudios de caso detallados, por medio del uso de marcos teóricos diferentes y alternativos.

Al revisionismo de las características tradicionales de la política exterior colombiana se suma René Ureña (2017), mediante el cuestionamiento de la vigencia, como valor retórico, del apego irrestricto de dicha política exterior al derecho internacional, aun cuando, desde el siglo XIX, este era percibido "como la puerta de entrada de las recientes repúblicas al club de naciones civilizadas" (Ureña, 2017, p. 65). La renuncia al pacto de Bogotá (Ureña, 2013), al igual que la intervención de la Corte Penal Internacional (CPI), en lo referente a la justicia transicional (Ureña y Acosta, 2015), son dos hechos recientes que soportan la tesis de Ureña. 
Esta relativización de derecho internacional en la política exterior de la nación colombiana puede obedecer a dos factores. Por una parte, a las transformaciones sociales propias de esta época, en donde el derecho internacional "se ha convertido en punta de lanza de reivindicaciones sociales muy controversiales en Colombia" (Ureña, 2017, p. 66); por otra, el derecho internacional actual no es un cuerpo unificado y homogéneo de normas, sistemas y procedimientos, de hecho, tal como señala Ureña (2017), se encuentra constituido por un conjunto de regímenes especializados: derechos humanos, derecho ambiental, derecho ambiental, entre otros, cada uno con formas de operar diferentes y a veces contradictorias entre sí.

\section{Política exterior colombiana: rupturas y continuidades}

Por un lado, en Colombia, se encuentran quienes consideran que, de un gobierno a otro, solo se presentaron cambios de forma, no de fondo, en la formulación y ejecución de la política exterior, es decir, hacia un estilo presidencial más moderado, prudente, "pragmático" o "realista" tanto en lo doméstico como en la proyección internacional y una atenuación apenas leve del alineamiento con EE.UU. (Pastrana y Vera, 2012, p. 58). Por otro lado, siendo una tendencia más fuerte, están quienes consideran la existencia de un giro rotundo, entre una administración y otra, manifestado en los siguientes aspectos, por mencionar los más relevantes: un nuevo acercamiento a los vecinos; el establecimiento de aliados como Perú, México y Chile; la diversificación de las relaciones con los Estados Unidos (Ramírez, 2011; Rojas, 2019); la participación activa en espacios multilaterales y organismos regionales, por medio de solicitud de ingreso a la OCDE (Vergara, 2012); el acercamiento a la APEC; la creación de la Alianza del Pacífico; la diversificación de la agenda internacional, y el uso de diferentes estrategias de inserción internacional, como la cooperación sur-sur (González, Mesa y Londoño, 2017), lo cual ha permitido ganar algunos márgenes de acción (Bermúdez, 2013).

Un tema que ha suscitado especial interés es la dicotomía securitización ${ }^{4}$ desecuritización, frente a la cual se reconocen dos posturas. Para una, no se

4 Securitización de la política exterior colombiana se refiere al proceso mediante el cual el tema de seguridad interna monopoliza la agenda internacional del país y subordina la política exterior a sus fines. La securitización procura, por una parte, lograr legitimidad ante la comunidad internacional y captar tanto recursos financieros como de cooperación. Por ende, por desecuritización se entiende un procedimiento de diversificación temática en la agenda y los objetivos de la política exterior, en donde la seguridad, si bien puede seguir jugando un rol representativo, ya no se considera la que determina la política exterior. 
ha presentado "desecuritización", sino que ha ocurrido un cambio parcial en la relación entre seguridad nacional y política exterior; se ha moderado el discurso, pero aún persiste una orientación antiterrorista: lo que se identifica aquí es una transformación de una securitización bilateral y personalista, con alta injerencia del ejecutivo, a una más diplomática y multilateral. La segunda orientación considera que entre ambos gobiernos se identifica un proceso de "desecuritización", dando espacio a una ampliación temática y de aliados en la inserción en espacios de diálogo multilateral y superación de las diferencias con los vecinos, especialmente con Ecuador y Venezuela (Crespo, 2012; Duarte, 2012). Así, se logra incorporar diversos temas en la agenda, dándole un carácter diversificado y cooperativo, redireccionando una política exterior que venía experimentando síntomas de rechazo y desgaste (Peréz y Rojas, 2012).

Ahora bien, es importante considerar que, en esta ruptura/continuidad que se pueda dar entre los gobiernos de Álvaro Uribe Vélez y Juan Manuel Santos, media un elemento clave: el diseño de una política de seguridad que, como se mencionó, generó un proceso de securitización de la ayuda internacional. El contexto colombiano, en su momento, luego del gobierno de Andrés Pastrana, con quien se inaugura el plan Colombia y se da paso a una mayor internacionalización de la agenda colombiana, da paso, luego de un fallido diálogo de paz con las Farc, a una visión de confrontación directa del gobierno colombiano con el grupo insurgente. Allí, es posible encontrar que la agenda interna de lucha contra el terrorismo se alinea con la internacional, particularmente norteamericana de lucha contra el terrorismo global, que afectó la estabilidad del orden mundial.

En parte, es comprensible este alineamiento con la agenda internacional y por ello la política exterior colombiana tiene una gran correspondencia con esta. El contexto posterior al 11 de septiembre de 2001 generó un cambio en los diseños de las agendas exteriores y Colombia no estuvo ajena a dicho fenómeno. Ahora bien, en la transición entre Uribe y Santos media también otro factor, esto es, el debilitamiento de las Farc y la consecución de una agenda de diálogo por parte del gobierno Santos con este grupo guerrillero. Es claro, para el gobierno nacional, que el debilitamiento de la insurgencia más antigua del continente es lo que posibilita llegar a una agenda de diálogo y, además, crea las condiciones para que la comunidad internacional apoye, de manera favorable, este proceso.

Por su parte, la investigación de Galeano, Badillo y Rodríguez (2019), bajo una orientación histórica y cualitativa, se centra en el análisis de la política exterior, entre el 2002 y el 2018, es decir, en la totalidad de las administraciones Uribe y Santos. Se hace énfasis en el comparativo entre ambas administraciones, puntualmente, tomando tres variables: la internacionalización del conflicto armado, las sociedades estratégicas del país en el contexto internacional y el servicio 
exterior. Concluyendo, con respecto a la variable de internacionalización del conflicto, en los periodos de gobierno de Álvaro Uribe, cuando la política exterior tomó tintes intermésticos, tal internacionalización del conflicto armado fue un medio para fundamentar la política de seguridad democrática en ese gobierno; en cambio, en las administraciones de Juan Manuel Santos, se observó un giro hacia una internacionalización de la paz.

En cuanto a las sociedades estratégicas, precisamente la internacionalización del conflicto armado agudizó los vínculos de dependencia con los Estados Unidos, durante el gobierno de Álvaro Uribe, generando una suerte de autoexclusión de algunas dinámicas regionales. En la era de Juan Manuel Santos, también en el marco de internacionalización de la paz y sin generar distanciamiento de Estados Unidos, se propició un acercamiento a diferentes actores, "que antes habían sido recelosos con respecto a la relación cercana entre Estados Unidos y Colombia (Unión Europea, Venezuela, Ecuador, Cuba, entre otros)" (Galeano, Badillo y Rodríguez, 2019, p. 74). Incluso, en lo tocante a la relación con el país norteamericano, se buscó, de forma bilateral, "que el país siguiera siendo considerado un aliado confiable, pero en el marco de unas relaciones bilaterales más equilibradas y con una agenda más diversificada" (Rojas, 2019, p. 14), por medio de la inclusión de temas que superara lo militar y estratégico: desarrollo social, crecimiento económico, medio ambiente, educación, energía y derechos humanos.

Con respecto a la variable de servicio exterior, se observa una similitud entre ambos gobiernos, a pesar de manifestaciones en torno a la necesidad de profesionalizar y dotar de mayor autonomía tanto a la cancillería como a ese servicio; este continuó siendo empleado de forma clientelar para pago de favores políticos.

\section{Perfil y características de la política exterior colombiana en las administraciones de Juan Manuel Santos}

El análisis y la caracterización de la política exterior del gobierno de Juan Manuel Santos es una línea de investigación bastante explorada. Bajo ella, se encuentra Sandra Borda con su artículo "Política exterior de la administración Santos: un Liderazgo de vía media" (2014), en el cual se considera que la nueva orientación en la política exterior colombiana obedece a un diagnóstico particular, con el objetivo de "limpiar" la imagen, en cierta medida, autoimpuesta de Colombia hacia el mundo. Esto responde, principalmente, a un eventual escenario de posconflicto, donde se debe "pensar el papel internacional del país fuera 
del marco de la guerra interna y de la guerra en contra del narcotráfico" (Borda, 2014, p. 38). Concluye Borda que el rol cada vez más activo y de potencia media regional que pretende desarrollar Colombia le puede imponer mayores responsabilidades en el sistema internacional, lo que podría caer en contradicción con la aún no resuelta y difícil situación interna de la nación colombiana.

Como es normal y necesario que suceda, una vez finalizada una administración presidencial, salen a la luz reflexiones e investigaciones, de carácter analítico, sobre alguna parcela de la administración saliente. Precisamente, cuando acaba la gestión Santos, se observa una creciente producción encaminada al análisis de la política exterior de ambos periodos de gobierno, por ejemplo, la de Sánchez y Campos (2019) y Ardila y Clemente (2019).

En la primera de estas investigaciones, se aborda un aspecto característico de la política exterior de dicho gobierno: la diplomacia por la paz, la cual estuvo dirigida hacia la búsqueda de apoyo interno y externo para consolidar el proceso de ese valor (Sánchez y Campos, 2019). El trabajo reconoce la complejidad de cada política exterior, el sesgo que puede recaer en los análisis que solo toman en cuenta un conjunto de variables; es por esto que selecciona tanto los factores sistémicos como los domésticos, con el fin de determinar el procedimiento de toma de decisión de esta estrategia de política exterior.

En procura del objetivo trazado, se establece un enfoque ecléctico que combina aspectos teóricos del realismo neoclásico, especialmente de los aportes de Lobell, Ripsman y Taliaferro (2009), y el enfoque cognitivo en política exterior de Jervis (1976), en lo que respecta a la percepción de amenazas. Con este sustento teórico que permite analizar la evidencia, se logra demostrar que, durante los años 2010-2018, en el nivel interno se alcanza un cambio en la percepción de amenazas; no solo en las guerrillas, como era tradicionalmente, sino también al incluir las bandas criminales (Bacrim) y fenómenos como la corrupción. En el ámbito sistémico, se concluye que el objetivo de la política exterior fue mejorar la imagen del país; esto, por medio de una diplomacia comercial y tanto del ingreso como de la participación activa en espacio multilaterales, no solo de carácter regional como la Alianza del Pacífico, sino también global, como la OCDE y la OTAN.

Por su parte, Ardila y Clemente (2019) realizan un análisis por fuera de las categorías respice polum-respice similia, que permite una consideración de las dimensiones sistémica, doméstica y personal, las cuales pueden incidir en el diseño y la práctica de la política exterior. Se afirma que, en materia de esta última, Juan Manuel Santos llevó a cabo una diplomacia tradicional "en permanente interacción con su política interna, con unas élites políticas y económicas 
fragmentadas frente a su proyecto principalmente político" (Ardila y Clemente, 2019 , p. 37), pero con algunas modificaciones, por ejemplo: la búsqueda de una diversificación temática y geográfica en el alcance de la política exterior; la estrategia hacia la internacionalización de la paz, acompañada de la búsqueda de una mejor imagen del país; la recuperación y reinserción del país a las dinámicas regionales; el otorgar mayor importancia a los espacios multilaterales y el mayor uso de la estrategia sur-sur.

También, el estudio mencionado permite vislumbrar el avance de nuevas temáticas y aspectos sobre política exterior, presentes en los ocho años del gobierno Santos: importancia creciente de las ciudades y regiones en el nivel internacional (paradiplomacia); necesidad de virar hacia una diplomacia científica; establecimiento de una política integral y de Estado en materia migratoria; instauración de un mayor énfasis en la región del Caribe. Sin embargo, el ejercicio de este tipo de diplomacia, también "basada en las dinámicas del Estado y del gobierno, como el clientelismo y la polarización de las élites internas, dificultaron una mirada más amplia con un lente conceptual más acorde con las necesidades del siglo XXI" (Ardila y Clemente, 2019, p. 46).

Estos análisis de la política exterior de la administración Santos conducen a la formulación de diversas preguntas, entre ellas: ¿Cuál fue la distancia existente, en materia de política exterior del gobierno Santos, entre la agenda inicial, la voluntad de llevarla a cabo y la capacidad real para hacerlo? Es la interrogante que busca responder Diana Rojas (2019), referida a cuál fue el margen de maniobra del gobierno colombiano para la implementación de la política exterior.

El análisis es realizado tomando en consideración dos aspectos puntuales de la agenda de política exterior de dicho gobierno: la búsqueda de apoyo internacional al proceso de negociación de la Habana y la ampliación de los vínculos externos. Con el fin de determinar el margen de maniobra en tales aspectos, son tomados tres factores: el respaldo político de actores tanto internos como externos, la capacidad institucional estatal, así como las dinámicas y tendencias dominantes en el nivel internacional. Rojas concluye que el gobierno Santos, en lo interno, contó con respaldo, al presentarse el proceso de paz como un proyecto político nacional, lo que a su vez permitió consolidar respaldo internacional. También, existió coordinación entre las instituciones estatales, con el fin de dar continuidad a las políticas durante el gobierno. Por último, se tuvo una visión política acorde con las tendencias internacionales predominantes. La conjunción de los tres factores permitió un alto grado de coherencia, traducido en un margen de maniobra suficiente para el logro de buena parte de los objetivos de política exterior. 


\section{La política exterior en un futuro inmediato: el posconflicto}

De forma directa e indirecta, la etapa posconflicto que atraviesa el país demanda y ocasiona cambios en las diferentes esferas del Estado. La política exterior no es ajena a este panorama. ¿Qué retos y oportunidades presenta la política exterior en el contexto de posconflicto? ¿Qué rol jurarán las fuerzas militares? ¿Cómo incide el posconflicto en la captación de recursos de cooperación internacional? Estas son algunas de las preguntas que pretenden dar respuesta a una serie de trabajos que, desde los últimos años, se abre paso en el campo de la política exterior colombiana.

A modo de un ejercicio prospectivo, Eduardo Pastrana y Diego Vera (2016), en "Política exterior colombiana, conflicto y posconflicto: algunas herramientas teórico-conceptuales para su análisis", "no sin antes advertir que la prospectiva política es mediada por posibles contingencias y un alto grado de incertidumbre" (Pastrana y Vera, 2016, p. 83), identifican retos que debe asumir la política exterior de Colombia articulada al posconflicto.

Uno de esos retos gira en torno a la cooperación internacional, en la cual Colombia, durante las dos últimas décadas, se constituyó en un receptor neto, a causa del conflicto armado. Ahora, la política exterior en un contexto de posconflicto tiene el desafío de articularse y apoyar la financiación de lo acordado en la Habana, "buscar apalancamiento externo a pesar de las restricciones de acceso a ayudas que le representa que Colombia sea calificada como país de renta media-alta" (Pastrana y Vera, 2016, p. 77), apreciación compartida por Duarte, González y Mesa (2017); es decir, captar la cooperación internacional asociada al discurso de viabilidad del posconflicto. Otro de los retos es articular los programas de impulso al sector agrario, base de los acuerdos, con la política de comercio exterior del país, donde los tratados comerciales firmados con países importadores de alimentos pueden jugar un rol importante y establecer acuerdos de cooperación agroindustrial. Finalmente, uno más de los retos se ve reflejado en la política de lucha contra la multicriminalidad organizada, que ha afectado de manera considerable a la región y exige la cooperación entre los Estados, para enfrentar los problemas de la región.

La lucha contrainsurgente y la contención de la amenaza interna, históricamente, demandaron una alta concentración de la capacidad del accionar de las fuerzas militares colombianas, en desmedro de la atención de ciertos asuntos, tales como fronteras, amenazas de otros Estados, cooperación militar, lucha contra el contrabando y la criminalidad transnacional. Precisamente, el posconflicto abre 
una ventana de oportunidades para una reorientación de las fuerzas militares, en procura de jugar un rol determinante en la política exterior del país. Trabajos como los de Bitar (2017) y Cancelado (2016) se orientan a la exploración de dichas oportunidades.

Sebastián Bitar, considera como oportunidades atender y hacer frente a los peligros potenciales externos para la seguridad nacional; utilizar, vía cooperación sur-sur, el conocimiento y la experiencia en seguridad, adquiridos durante décadas de lucha contra la insurgencia y el narcotráfico, para capacitar a fuerzas militares de Estados que presenten estas problemáticas. A ello se puede adicionar la posibilidad de transitar de una fuerza contrainsurgente hacia una multimisión, participando de forma activa en misiones internacionales de paz (Cancelado, 2016; González y Mesa, 2018).

\section{Las instituciones estatales y actores no gubernamentales en la política exterior colombiana}

Bajo la óptica de los actores y determinantes internos, se encuentran trabajos que abordan tanto a las instituciones estatales como a los entes no gubernamentales. En cuanto a ambos tipos de actores, quizás la investigadora más activa ha sido Martha Ardila (2008, 2009 y 2009b), permitiendo ampliar el análisis con diferentes variables, unidades de análisis y referentes teóricos, como se destaca a continuación.

En Actores no gubernamentales y política exterior. A propósito del sector académico y el diseño de la política exterior migratoria colombiana (2009), Marta Ardila parte del diagnóstico de la poca participación que, a la hora de formular la política exterior, se presenta por parte de los actores no gubernamentales en Colombia; a pesar de que las tecnologías de la información y comunicación han facilitado la intervención de este tipo de actores en los asuntos internacionales. A lo anterior se suma Vargas (2014), quien considera que, pese a la asimilación como actores de alta importancia decisoria de aquellos no estatales en el nivel del sistema internacional, en el caso colombiano, aún no se evidencia una presencia constante e institucionalizada de ellos en el proceso de la política exterior. Incluso, actores no estatales que podrían contar con canales de comunicación más estrechos con el gobierno de turno, como los partidos políticos, "tienden a ser invitados de piedra en la construcción y puesta en marcha de la política exterior. Sus posiciones son oídas por el Ejecutivo, pero no vinculantes" (Barrero y Niño, 2012, p. 228). 
Retomando a Ardila (2009), emplea como método el estudio de un único caso: la influencia del sector académico para el diseño de la política migratoria. El texto, por medio de sus conclusiones, permite identificar aportes teóricos a la presente propuesta, por ejemplo: que el Estado ya no es el único actor de las relaciones internacionales y que hay muchos otros actores no gubernamentales, los cuales, desde diferente posición, inciden en la formulación de los lineamientos internacionales (Ardila, 2009, p. 121). No obstante, en Colombia, dicha participación es intermitente y depende del criterio de los tomadores de decisiones en política exterior.

Con respecto al primer grupo referido a las instituciones estatales, se destaca Anatomía de un actor: el ministerio de Defensa Nacional y la política exterior (Ramírez, 2012), en el cual se determina el rol y la importancia que tiene este ministerio, en el marco de una nueva política exterior compleja y, por lo tanto, en el que confluyen cada vez más actores. Este aspecto se caracteriza por brindar información de inteligencia necesaria para toma de decisiones exteriores y materia de seguridad.

También, Ardila, en El Congreso y la política exterior colombiana. A propósito de la comisión segunda (2008), haciendo uso de fuentes primarias como documentos oficiales y entrevistas, explora el vínculo entre la comisión segunda del Congreso, encargada de los asuntos internacionales en el legislativo, y la política exterior colombiana. Tres elementos ayudan a entender dicha relación: 1) tanto el marco como las herramientas de la constitución política de 1991, 2) las actividades que desarrolla la comisión y 3) las características generales de la política exterior del país.

Por medio de un recorrido normativo de la constitución política de 1991, la autora identifica atribuciones, funciones, obligaciones y alcance del Congreso, especialmente, de la comisión segunda, en materia de política exterior. Como hallazgo de este trabajo, sobresalen las acciones de control político que, acerca de la agenda internacional, ha realizado la comisión, debilitando así la tradicional afirmación reduccionista, la cual encasilla la política exterior del país (de un carácter netamente personalista). Asimismo, se concluye que debe existir mayor capacitación en temas internacionales, por parte de los integrantes de la comisión, y establecer sinergias con la Cancillería, para lograr un papel más activo en la toma de decisiones.

Entre las investigaciones más recientes, se encuentra Las élites y la política exterior colombiana (1958-2010) (Puyo, 2017), que emplea un enfoque tanto interméstico como constructivista, al igual que se apoya en los estudios sobre élites y toma de decisión. Esto, con la intención, así como esta propuesta de 
tesis, de superar categorías reduccionistas en las cuales ha sido enfrascada la política exterior colombiana, como el respice polum, el respeto irrestricto al derecho internacional y el bajo perfil; se busca, ampliar la discusión, la inclusión de variables y una mayor profundidad de esta política. La política exterior es sumida no como resultado de coyunturas externas, sino de relaciones de poder de los actores al interior del país, particularmente, las élites.

Un aporte novedoso, que puede abrir línea para futuras investigaciones, es el clasificar en cuatro los tipos de élites que inciden la política exterior colombiana. La primera es la élite de tipo político, asociada "a la pertenencia a partidos políticos [...] a las relaciones construidas en el nivel local y nacional. Al desempeño de cargos públicos [...] y a las tradiciones políticas familiares" (Puyo, 2017, p. 161). La segunda, es la élite de tipo técnico, a razón de la formación académica y del recorrido profesional, en el campo de la diplomacia y de las relaciones internacionales. En tercer lugar, se encuentra la élite del sector privado, no siempre vinculada directamente a un partido político o con formación académica específica, pero útil en procesos de negociación, tratados comerciales y acuerdos de integración económica. Finalmente, la cuarta élite la conforma la de origen regional, vinculada, precisamente, a los poderes y familias tradicionales de ciertas regiones del país. Según los hallazgos, en el periodo analizado, la élite técnica, a pesar de ser minoría, es más propositiva, independiente y dinámica, al momento del diseño y de la ejecución de la política exterior, a causa de la experiencia y la formación profesional propia del campo diplomático.

"El papel de los partidos políticos en la política exterior colombiana" (Barrero y Niño, 2012) parte también del reconocimiento de la poca literatura dedicada a explorar los determinantes internos de la política exterior del país. A modo de indagación y con el constructivismo como orientación teórica, caracteriza el marco institucional de la construcción y ejecución de la política exterior colombiana, determinando el alto contenido presidencialista de esta. El texto concluye, entre otras cosas, que a causa del gran presidencialismo del sistema político colombiano y a pesar de que los partidos incluyan en sus plataformas ideológicas temas internacionales, es casi nula la presencia que puedan ejercer justo los partidos políticos en la formulación de la política exterior.

En este sentido, la debilidad de la presencia de un actor como los partidos es irrelevante a la hora de considerar una definición de interés nacional; son una agrupación que, si bien tiene peso político, no se pone de manifiesto en la formulación de la política exterior nacional ni mucho menos genera reflexiones de corte político que permitan proyectar en materia internacional al propio Estado. 
En cuanto a las organizaciones no gubernamentales, se destacan las investigaciones de Davide Bocchi $(2012,2013)$. La primera de estas, "El papel de las organizaciones no gubernamentales en la política exterior colombiana", parte del reconocimiento y de la importancia, que, en el marco de la globalización, han tomado este tipo de actores. Su influencia, generalmente vía presión mediática o movilización, hacia la política exterior en Colombia se presenta para los temas de derechos humanos, cooperación, salida negociada al conflicto armado. El autor concluye que, en la construcción de la política exterior, las sugerencias de las ONG no fueron atendidas por el gobierno de Juan Manuel Santos y, en consecuencia, se presenta una "diplomacia paralela" a aquella. Pero ¿qué implicación trajo consigo la no inclusión de las sugerencias de las ONG en la política exterior colombiana? Se argumenta que este hecho dificultó la pretensión del gobierno Santos de lograr una mejor posición en el nivel internacional y de mostrarse como un país con cierto liderazgo regional: “[...] son los problemas internos que las ONG nacionales e internacionales evidencian y transmiten al exterior, impidiendo que Colombia logre posicionar la imagen de sí que quisiera en la escena internacional [...]" (Bocchi, 2013, p. 280).

Una de las consideraciones tradicionales sobre la política exterior colombiana es que, a causa de ser un proceso decisorio hermético, es poca la importancia que la opinión pública asume sobre estas temáticas; situación diferente a lo que ocurre con otras políticas públicas, donde se observa una mayor movilización social y atención mediática. Este desentendimiento hacia la política exterior refuerza, a su vez, el carácter hermético en el proceso decisorio, cubriendo de un velo de secreto y misterio los asuntos externos del país y volviéndose así una suerte de circulo vicioso.

En este sentido, en los textos de Diasabá y Rueda (2012) y González (2012), se explora tanto la importancia como la cobertura que los medios de comunicación nacional le otorgan a la política exterior del país. Por medio de entrevistas a expertos y revisión documental de periódicos, se considera que la prensa y los medios de comunicación en el país dan muy poca cobertura e importancia a los asuntos internacionales, en especial a la política exterior de la nación colombiana. Dicha cobertura se limita a la descripción de hechos y no a un análisis profundo y objetivo, en buena medida, a causa de la carencia de profesionales de la comunicación con formación específica en relaciones internacionales (González, 2012) y a la existencia de "temor a ser "antipatriota", lo que genera que la prensa sea poco crítica de la política exterior del gobierno respectivo" (Diasabá y Rueda, 2012, p. 253). 


\section{Conclusiones}

La revisión de la literatura especializada en la política exterior colombiana ha permitido identificar las diferentes tendencias en torno al objeto de estudio planteado. Esto incluye la existencia de un consenso casi generalizado de que, efectivamente, la política exterior del gobierno Santos, en mayor o menor grado, experimentó cambios con respecto a su antecesor. Tal situación se evidencia en la diversificación de la agenda; la búsqueda de reintegrarse a las dinámicas regionales y el multilateralismo expresado en participación activa en espacios regionales y globales; el tratamiento diferencial, dado el relacionamiento con los Estados Unidos, entre otros. Sin embargo, también se identificaron líneas de continuidad tales como la clientelización del servicio exterior y la necesidad de reformarla, en beneficio de la proyección internacional del país.

Esta revisión de la literatura especializada en el análisis de la política exterior ha contado con varios propósitos: determinar las tendencias actuales de investigación sobre la temática particular; identificar los métodos y las orientaciones teóricas empleadas para los análisis; determinar posibles vacíos investigativos, y conocer el nivel de actividad de esta línea de indagación.

La metáfora de la caja negra tradicionalmente ha estado presente a la hora de explicar y caracterizar la política exterior del país; es decir, no se explora en su interior, considerándola como algo dado, sea por el líder de turno, como acomodación al contexto internacional (especialmente a la política exterior norteamericana) o como respuesta de la situación particular y conflictiva de Colombia. No obstante, en los últimos años, han surgido iniciativas académicas que propenden por ampliar los márgenes de análisis y variables de estudio de esta política. Particularmente, se han realizado estudios sobre partidos políticos, medios de comunicaciones, algunas instituciones estatales y ONG. Por lo tanto, los actores con incidencia e interés en la política exterior del país son un tema con una alta riqueza potencial. Si bien hay un camino recorrido en este sentido, gracias a las investigaciones mencionadas en este espacio, al ser un campo de estudio amplio, el impulso académico se debe aprovechar, profundizando y ampliando el examen de diferentes actores como grupos económicos, medios de comunicación, agrupaciones sociales, organizaciones y regímenes internacionales. Otro campo general, que cuenta con un importante potencial investigativo, son los posibles determinantes internos, tales como la cultura estratégica, el diseño institucional, el proceso de toma de decisiones, el rol de las percepciones, la identidad y la historia.

También, en los últimos años, se puede evidenciar el progresivo abandono o difuminación, en los análisis de política exterior colombiana, de las categorías 
respice polum-respice similia, que, desde la década de 1980 estuvieron presentes en estos análisis. Lo anterior, en beneficio del uso de conceptos, con un mayor desarrollo y riqueza teórica. En este sentido, han ganado espacio el uso de orientaciones teóricas como el realismo neoclásico o el constructivismo y un retorno al Foreign Policy Analysis (FPA), pero también aportes desarrollados en América Latina como el realismo periférico y la autonomía relacional. Esto ha permitido disposiciones teórico-metodológicas eclécticas, en las cuales se combinan varios cuerpos teóricos para el análisis de situaciones específicas, sin separar las dimensiones interna-externa, sino procurando una visión holística.

En suma, los estudios de política exterior colombiana actualmente son una línea de investigación dinámica y activa. Esta ha superado la etapa de la descripción y generalizaciones, en procura de lograr una mayor profundidad en los factores explicativos que le dan forma. Por lo tanto, de continuar estas tendencias, se vislumbra un futuro inmediato de una consolidación investigativa.

Lo anterior coincide con un mayor nivel de autonomía y desarrollo disciplinar del estudio de las relaciones internacionales en Colombia. En décadas pasadas, los estudios internacionales se encontraban directamente vinculados a las facultades de derecho, por lo que imperaba una visión legalista en sus análisis (Cardona, 1992) y, posteriormente, a los programas de ciencia política. Este proceso de mayor autonomía y desarrollo disciplinar está especialmente jalonado por la creación de departamentos y pregrados de relaciones internacionales (en varias universidades del país, grupos de investigación, redes especializadas, eventos académicos, revistas científicas), así como por la formación en los niveles de maestría y doctorado.

\section{Referencias}

Amaya, R. (2017). Las propiedades de la política exterior colombiana: repensando lo que sabemos del accionar externo del país. En A. Tickner y S. Bitar (eds.), Nuevos enfoques para el estudio de las relaciones internacionales de Colombia (pp. 39-64). Bogotá: Universidad de los Andes.

Ardila, M. (2009). Actores no gubernamentales y política exterior. A propósito del sector académico y el diseño de la política exterior migratoria colombiana. Colombia Internacional, (69), 108-123.

Ardila, M. y Clemente, I. (2019). Santos: una diplomacia tradicional con cambios. Oasis, (29), 29-56. Doi: https://doi.org/10.18601/16577558.n29.03

Ardila, M.; Cardona, D. y Ramírez, S. (2005). Colombia y su política exterior en el siglo XXI. Bogotá: Friedrich Eber Stiftung Colombia. 
Ardila, M.; Carvajal, L.; Garay, J.; Marín, M.; Niño, J. y Puyana, J. (2008). La toma de decisiones de la política exterior colombiana. Bogotá: Universidad Externado.

Ardila, M.; Montilla, P. y Garay, J. (2009b). Actores no estatales y política exterior colombiana: casos de los sectores académicos y empresariales. Bogotá: Universidad Externado.

Barrero, F. y Niño, C. (2012). El papel de los partidos políticos en la política exterior colombiana. En S. Jost (ed.), Colombia: ¿una potencia en desarrollo? Escenarios y desafíos para su política exterior (pp. 227-247). Bogotá: Konrad Adenauer.

Bermúdez, C. (2013). La política exterior colombiana en el marco de la integración latinoamericana. Analecta, 3(4), 67-91.

Bernal, J. y Tickner, A. (2017). Imaginario de política exterior y proamericanismo en Colombia. En A. Tickner y S. Bitar (eds.), Nuevos enfoques para el estudio de las relaciones internacionales de Colombia (pp. 3-38). Bogotá: Universidad de los Andes.

Bitar, S. (2017). Las fuerzas armadas en el posconflicto: una renovada preocupación por lo internacional. En A. Tickner y S. Bitar (eds.), Nuevos enfoques para el estudio de las relaciones internacionales de Colombia (pp. 193-212). Bogotá: Universidad de los Andes.

Bocchi, D. (2012). El papel de las organizaciones no gubernamentales en la política exterior colombiana. En S. Jost (ed.), Colombia: ¿una potencia en desarrollo? Escenarios y desafíos para su política exterior (pp. 283-299). Bogotá: Konrad Adenauer.

Bocchi, D. (2013). La política exterior colombiana y el costo de ignorar a las organizaciones no gubernamentales. Papel Político, 18(1), 261-284.

Borda, S. (2014). Política exterior de la administración Santos: un liderazgo de vía media para Colombia. En A. Ayuso; S. Borda; F. Floriano; V. Moy y M. Pallarès (eds.), Liderazgos regionales emergentes en América Latina (pp. 29-39). Barcelona: CIDOB.

Cancelado, H. (2016). La política exterior y el nuevo rol de las FF.AA. en el contexto de la nueva estrategia y doctrina de defensa con miras al posconflicto. En E. Pastrana y H. Gehring (eds.), Política exterior colombiana. Escenarios y desafios en el posconflicto (pp. 155-178). Bogotá: Pontificia Universidad Javeriana. 
Cardona, D. (1992). Los estudios sobre política exterior colombiana: supuestos y temas en varios autores representativos. En R. Rusell, Enfoques teóricos y metodológicos para el estudio de la política exterior (pp. 109-142). Buenos Aires: Grupo editor latinoamericano.

Crespo, R. (2012). Análisis de política exterior en Colombia: gobierno de Juan Manuel Santos. ¿Continuidad de un proceso o cambio de rumbo? Equidad $y$ desarrollo, (17), 149-175.

Diasabá, V. y Rueda, C. (2012). La política exterior colombiana en los medios de comunicación. En S. Jost (ed.), Colombia: ¿una potencia en desarrollo? Escenarios y desafíos para su política exterior (pp. 251-264). Bogotá: Konrad Adenauer.

Drekonja, G. (1983). Retos de la política exterior colombiana. Bogotá: Cerec.

Duarte, J. (2012). La política exterior de Colombia con la Unión Europea: cambios y continuidades a partir de una nueva Colombia. En S. Jost (ed.), Colombia: ¿una potencia en deasrrollo? Escenarios y desafios para su politica exterior (pp. 339-355). Bogotá: Konrad Adenauer.

Duarte, L.; González, C. y Mesa, J. (2017). Retos de la cooperación internacional para el desarrollo en Colombia. Estudios Internacionales, 49 (188), 111-146.

Galeano, H.; Badillo, R. y Rodríguez, M. (2019). Evolución de la política exterior de Colombia en el periodo 2002-2018. Oasis, (29), 57-79.

Gómez, M.; Galeano, C. y Jaramillo, D. (2015). El Estado del arte: una metodología de investigación. Revista Colombiana de Ciencias Sociales, 6(2), 423-442.

González, C. H.; Mesa, J. C. y Londoño, G. A. (2017). Política exterior colombiana 2010-2014: ¿giro a la autonomía? Relaciones Internacionales, Estrategia y Seguridad, 12(1), 267-291.

González, J. (2012). La prensa, ¿convidada de piedra en el examen de la política exterior? En S. Jost (ed.), Colombia: ¿una potencia en desarrollo? Escenarios y desafíos para su política exterior (pp. 265-282). Bogotá: Konrad Adenauer.

González, R. (2004). La política exterior de Colombia a finales del siglo XX. Primera aproximación. Investigación \& Desarrollo, 12(2), 258-285.

González, R.; Galeano, H. y Trejos, L. (2015). Estados Unidos en la política exterior colombiana: ¿Aliado incondicional? Económicas CUC, 36(1), 43-56.

González, C. y Mesa, J. (2018). Incidencia del narcotráfico en la formulación y ejecución de la política exterior colombiana. Escenarios, empresa y territorio $7(10), 81-100$. 
Jervis, R. (1976). Perception and Misperception in International Politics. New Jersey: Princeton University Press.

Lobell, S.; Ripsman, N. y Taliaferro, J. (2009). Neoclassical Realism, the state and foreign policy. New York: Cambridge University Press.

Pardo, R. y Tokatlian, J. (1988). Política exterior colombiana: ¿de la subordinación a la autonomía? Bogotá: Tercer mundo editores.

Pastrana, E. (2011). La política exterior colombiana y la percepción de los expertos: Cambio o continuidad en la era Santos. KAS Paper, (15), 1-27.

Pastrana, E. y Vera, D. (2012). De uribe a Santos: ¿continuidad o nueva orientación de la política exterior colombiana? En S. Jost (ed.), Colombia: ¿una potencia en desarrollo? Escenarios y desafios para su política exterior (pp. 57-80). Bogotá: Konrad Adenauer.

Pastrana, E. y Vera, D. (2016). Política exterior colombiana, conflicto y posconflicto: algunas herramientas teórico-conceptuales para su análisis. En E. Pastrana y H. Gehring (eds.), Politica exterior colombiana: escenarios y desafios en el posconflicto (pp. 23-60). Bogotá: Editorial Pontificia Universidad Javeriana.

Peréz, M. y Rojas, E. (2012). Debates y perspectivas de la política exterior colombiana en la era Santos. La Plata: Universidad Nacional de la Plata. VI Congreso de Relaciones Internacionales.

Puyo, G. (2017). Las élites y la política exterior colombiana (1958-2010). Bogotá: Universidad Nacional de Colombia.

Ramírez, C. (2012). Anatomía de un actor: el Ministerio de Defensa Nacional y política exterior colombiana. En S. Jost (ed.), Colombia: ¿una potencia en desarrollo? Escenarios y desafios para su política exterior (pp. 211-226). Bogotá: Konrad Adenauer.

Ramírez, S. (2011). El giro de la política exterior colombiana. Nueva sociedad, (231), 79-95.

Retamozo, M. (2014). ¿Cómo hacer un proyecto de tesis doctoral en Ciencias Sociales? Ciencia, Docencia y Tecnología, 25(48), 173-202.

Rojas, D. (2019). La política internacional de la administración Santos: entre los imperativos y las aspiraciones. Oasis, (29), 7-27. Doi: https://doi. org/10.18601/16577558.n29.02

Sánchez, F. y Campos, S. (2019). La política exterior de Santos: estrategia y diplomacia por la paz. Oasis, (29), 81-104.

Tickner, A. (2003). En busca de Aliados para la "seguridad democrática": la política exterior del primer año de la administracion Uribe. Colombia Internacional, (56-57), 64-81. 
Tickner, A. (2007). Intervención por invitación. Claves de la política exterior colombiana y de sus debilidades principales. Colombia Internacional, (65), 90-111.

Tickner, A. y Borda, S. (2011). Relaciones Internacionales y politica exterior de Colombia. Bogotá: Universidad de los Andes.

Tickner, A. y Borda, S. (2011). Las Relaciones Internacionales en Colombia. Creación, consolidación y producción disciplinar. En S. Borda y A. Tickner (comps.), Relaciones Internacionales y política exterior de Colombia (pp. 21-47). Bogotá: Ediciones Uniandes.

Tokatlian, J. (2000). La mirada de la política exterior de Colombia ante un nuevo milenio: ¿ceguera, miopía o estrabismo? Colombia Internacional, (48), 35-43.

Urueña, R. (2013). Colombia se retira del pacto de Bogotá. Causas y efectos. Anuario de Derecho Público, (1), 511-547.

Urueña, R. (2017). La crisis del "club de caballeros": una reconsideración del respeto al derecho internacional como característica de la política exterior colombiana. En A. Tickner y S. Bitar, Nuevos enfoques para el estudio de las relaciones internacionales de Colombia (pp. 65-89). Bogotá: Universidad de los Andes. Doi: http://dx.doi.org/10.7440/2017.45.

Urueña, R. y Acosta, D. (2015). Beyond Justice, Beyond Peace? Colombia, the Interests of Justice, and the Limits of International Criminal Law. Criminal Law Forum (26), 291-318. Doi: http://10.1007/s10609-015-9248-1

Vargas, L. (2014). Actores no estatales y política exterior: una revisión preliminar del caso colombiano. Revista Análisis Internacional, 5(1), 189-206.

Vergara, R. (2012). Análisis de política exterior en Colombia: gobierno de Juan Manuel Santos, ¿continuación de un proceso o cambio de rumbo? Equidady desarrollo, (17), 149-175. 
